\title{
Diversidad de haplotipos del complejo principal de histocompatibilidad en equinos de la raza Árabe de la República Argentina
}

\author{
Major histocompatibility complex haplotype diversity in \\ Arab horses from Argentina
}

\author{
Sadaba SA ${ }^{1,2^{*}}$, Corbi Botto $\mathrm{CM}^{1,2}$, Zappa ME${ }^{1}$, Carino $\mathrm{MH}^{1}$, \\ Villegas Castagnasso EE¹, Peral García P1', Díaz S1
}

${ }^{1}$ Instituto de Genética Veterinaria "Ing. Fernando Noel Dulout" (IGEVET). Facultad de Ciencias Veterinarias, Universidad Nacional de La Plata. CCT La Plata - CONICET. La Plata, Argentina.

${ }^{2}$ Becarios de Consejo Nacional de Investigaciones Científicas y Técnicas (CONICET)

${ }^{*}$ Correo electrónico del autor: ssadaba@fcv.unlp.edu.ar

Resumen: La diversidad de los genes del complejo principal de histocompatibilidad (MHC) es clave en su función en la presentación de antígenos en el sistema inmune. La tipificación indirecta basada en microsatélites (STR) del MHC aporta información de la variabilidad genética y de la estructura poblacional y es una estrategia para conocer procesos selectivos y evolutivos. Con el fin de caracterizar una población de caballos de raza Árabe, se identificaron los haplotipos del MHC sobre la base de tres microsatélites (UM011, DRB2-STR2 y COR112) abarcando una región de $1 \mathrm{Mpb}$. EI ADN genómico se extrajo de sangre y pelo de 30 caballos de cinco haras de la provincia de Buenos Aires y los STRs se amplificaron con cebadores fluorescentes para tipificar en secuenciador automático. Se estimaron parámetros poblacionales y de diversidad y la detección de haplotipos se realizó mediante análisis de segregación y con el programa de reconstrucción de haplotipos PHASE. Se reconocieron 24 haplotipos; entre ellos, 12 se verificaron por segregación en los registros de pedigree del Stud Book. Los resultados obtenidos demostraron la existencia de ligamiento con alelos conocidos de genes de clase II del equine leukocyte antigen (ELA) y la cantidad de haplotipos identificada permitió expandir la valoración de la diversidad del MHC equino. Esta metodología constituye una herramienta de utilidad y un método alternativo para la tipificación del MHC en linajes de caballos y en estudios poblacionales relacionados con la inmunidad.

Palabras clave: Haplotipos; complejo principal de histocompatibilidad; equinos Árabes

\begin{abstract}
Genetic diversity of the major histocompatibility complex ( $\mathrm{MHC})$ is essential for the antigen recognition and presentation during the immune response. Indirect $\mathrm{MHC}$ typing by microsatellites (STR) provides data on genetic diversity and population structure, and provides knowledge of selective and evolutionary processes. In order to characterize a sample of Arab horses, we identified $\mathrm{MHC}$ haplotypes based on three STRs (UM011, DRB2-STR2 and COR112) covering a region of $\sim 1 \mathrm{Mpb}$. Genomic DNA was extracted from blood and hair of 30 horses from five farms of the province of Buenos Aires. STRs were amplified with fluorescent primers and typed in an automated sequencer. Population and diversity parameters were estimated. Haplotype detection was performed by segregation analysis and with the PHASE program for reconstructing haplotypes. Of the 24 haplotypes identified, 12 were verified by segregation in the Stud Book pedigree records. Our results showed linkage with known equine leukocyte antigen (ELA) class II alleles. The number of haplotypes identified allowed to expand the estimates of diversity in the equine MHC. This methodology is a useful tool and an alternative approach to type $\mathrm{MHC}$ in horse lineages and immune-related population studies.
\end{abstract}

Key words: Haplotypes; major histocompatibility complex; Arab horses 


\section{Introducción}

En los vertebrados, el complejo principal de histocompatibilidad (MHC) se localiza en una región cromosómica y está constituido por una familia multigénica, cuyos productos cumplen funciones importantes en la respuesta inmune (Lewin et al., 1999). Estos genes pueden dividirse en tres clases principales: clase I, clase II y clase III. EI MHC del caballo se conoce como equine leukocyte antigen (ELA) y esta región de genes estrechamente ligados es responsable de la presentación de antígenos propios y no propios al sistema inmune y así es fundamental en el reconocimiento y regulación de la respuesta inmune (Figura 1). Las moléculas del MHC cumplen estos roles uniéndose y presentando péptidos antigénicos a los linfocitos $\mathrm{T}$, pudiendo conducir a varios eventos, incluyendo la eliminación de células infectadas o neoplásicas o el rechazo celular a órganos o tejidos trasplantados (Ansari et al., 1988).

En el MHC existen combinaciones características de alelos de diferentes loci (haplotipos) con frecuencias elevadas, algunos de los que exhiben desequilibrio de ligamiento en diferentes grupos étnicos y en especies de animales domésticos (Hedrick, 1994). El número de haplotipos también varía entre especies y aún dentro de especies, elevando la variabilidad del sistema. También existen variaciones en el número de genes que componen cada haplotipo (Chardon et al., 1999; Ellis \& Ballingal, 1999).

La alta diversidad de los genes de clase I y clase II del MHC de los vertebrados es un rasgo clave en la función que desempeñan en la presentación de antígenos en el sistema inmune (Hughes \& Nei ,1992). Sin embargo, en equinos, la asignación de haplotipos del $\mathrm{MHC}$ a individuos continua siendo un reto a pesar de los avances tecnológicos y del conocimiento del genoma equino. El principal reporte en caballo doméstico corresponde a los haplotipos de los genes de clase II (Tseng et al., 2010), basados inicialmente en los talleres internacionales establecidos para identificar las especificidades serológicas para el ELA desde la década de 1980 (Lazary et al. 1985, 1988). La falta de descripciones completas de haplotipos equinos del $\mathrm{MHC}$ de clase II y la falta de métodos específicos para haplotipos como en otras especies, determinan que la genotipificación basada en microsatélites del MHC (sequence tandem repeat - STR) sea de utilidad para la tipificación indirecta (Bastos-Silveira et al., 2009; Kalemkerian et al., 2011; Santucci et al., 2007; Wagner et al., 1996) y también son valiosos para estudiar linajes y divergencia de los haplotipos (Brinkmeyer-Langford et al., 2013).

Dado que utiliza microsatélites localizados dentro de o cercanos a genes candidatos de interés, pueden, además, aportar información acerca de la variabilidad genética y la estructura de la población, y puede ser también una estrategia valiosa para conocer los procesos de selección y evolutivos relacionados con la historia de las razas equinas. Para los propósitos de tipificación, los microsatélites también son herramientas valiosas porque exhiben numersos alelos (polimorfismo) y elevada heterocigosis (Meagher \& Potts, 1997; Tseng et al., 2010). Tseng et al. (2010) demostraron que múltiples haplotipos de microsatélites pueden asociarse con un único serotipo, observando una mayor resolución, sensibilidad y precisión de la genotipificación de ADN en comparación con métodos serológicos (Azor et al., 2007; Brinkmeyer-Langford et al., 2013; Tseng et al., 2010). Además, los estudios genéticos poblacionales del polimorfismo del MHC en numerosas especies, principalmente en humanos, especies domésticas y de laboratorio, han conducido a identificar asociaciones con enfermedades que vinculan determinados genotipos del MHC con susceptibilidad o resistencia a varias condiciones patológicas (Maillard et al., 2002; Meredith et al., 1986; Sharif et al., 1998; Swinburne et al., 2012).

El objetivo de este trabajo consistió en determinar la diversidad genética de tres marcadores de tipo microsatélite localizados dentro de la región del ELA. Estos marcadores serán utilizados para identificar, de forma preliminar, la composición haplotípica en grupos familiares de caballos de raza Árabe y definir su poder de discriminación para la identificación de haplotipos sobre la base del desequilibrio de ligamiento característico del $\mathrm{MHC}$.

\section{Materiales y métodos Muestras y extracción de ADN}

Para el análisis experimental se utilizaron un total de 30 caballos de la raza Árabe. Los caballos pertenecen a tres grupos familiares, de diferentes haras de la provincia de Buenos Aires. Se registraron los datos de identificación y genealógicos de cada caballo. Todos se encontraban registrados en el Stud BookArgentino. Las muestras de sangre se tomaron por punción yugular en condiciones de esterilidad y con anticoagulante (EDTA $6 \%$ ) y se refrigeraron hasta la extracción de ADN. Los pelos fueron arrancados de la cola y se almacenaron en sobres individuales, adecuadamente rotulados. El ADN genómico se extrajo a partir de sangre periférica utilizando la técnica de DNAzol (Gibco, BRL-Life Technologies, Rockville, MD, USA -Cox, 1968-), según las recomendaciones del fabricante. El ADN genómico de los bulbos pilosos se extrajo mediante la técnica de extracción con $\mathrm{NaOH}$ 0,2 M. El ADN purificado se cuantificó en espectrofotómetro NanoVue (GE Healthcare), se visualizó en geles de agarosa al $1 \%$ p/v y se usó como molde para la reacción de amplificación mediante el método de reacción en cadena de la polimerasa (PCR). 


\section{Genotipificación de los marcadores STRS}

La amplificación de los STRs se realizó por separado para cada uno de ellos por medio de reacción en cadena de la polimerasa (PCR) utilizando cebadores oligonucleótidos específicos para cada uno de los STRs (COR112-F 5'-TTACCTGGTTATTGGTTATTTGG-3'; COR112-R5'-TCACCCACTAAATCTCAAATCC-3'; UM011-F 5'-TGAAAGTAGAAAGGGATGTGG3'; 5'-TCTCAGAGCAGAAGTCCCTG3'; DRB2 STR2F5'-AAGGACGTCCTGGATGACGC-3'; Eca-DRBSTR1-2R 5'AGTTACAAGGGACCGTGTGA3'). Los cebadores forward de cada par se marcaron con el colorante fluorescente FAM y se amplificaron en un reactor MAXYGENE (Axygenelnc, Union City, CA) para la posterior identificación mediante análisis de fragmentos. Las condiciones de ciclado consistieron en desnaturalización inicial a $95^{\circ} \mathrm{C}$ por $5 \mathrm{~min}, 35$ ciclos a $94^{\circ} \mathrm{C}$ por $1 \mathrm{~min}, 55^{\circ} \mathrm{C}$ por $30 \mathrm{~s}$ y $72^{\circ} \mathrm{C}$ por $30 \mathrm{~s}$. La extensión final se realizó a $72{ }^{\circ} \mathrm{C}$ por $15 \mathrm{~min}$. Los fragmentos de PCR se discriminaron en secuenciador automático MegaBase1000 (GE Healthcare, Buckinghamshire, UK) usando ET550-Rox como estándar de peso molecular (GE Healthcare). Los genotipos se asignaron utilizando el programa FragmentProfiler Software Suit version 2.2 (MegaBase Build 1.2.0311.2500, Amersham Biosciences, GE Healthcare, Buckingamshire, UK, 2003).

\section{Análisis estadístico y detección de los haplotipos de clase II del ELA}

Para cada marcador se estimaron las frecuencias alélicas y genotípicas, la heterocigosis esperada (He) y observada (ho), número de alelos (na) y el equilibrio de HW (EHW), mediante el test de estimación de probabilidad de $p$-valores utilizando el programa GENEPOP 4.0 (ftp://ftp.cefe.cnrsmop.fr/genepop/ Rousset 2008). El desequilibrio de ligamiento entre los tres marcadores STRs se estimó también mediante el programa Genepop. La detección de haplotipos se realizó con el programa de reconstrucción de haplotipos
PHASE y se verificó mediante análisis de segregación de acuerdo con los registros del Stud Book Argentino.

\section{Resultados \\ Localización genómica de los STRs}

Se seleccionaron los marcadores STRs UM011 (Meyer et al. 1997), COR112 (Tseng et al., 2010) teniendo en cuenta reportes previos acerca de su ligamiento con genes de clase II del ELA, y ELA-DRB2 (STR2), desarrollado en este trabajo. Para identificar la localización de los loci STRs se realizaron búsquedas BLAST (https://blast.ncbi.nlm.nih.gov/Blast.cgi) enfrentando la secuencia genómica de referencia en el cromosoma Eca20. Se localizó UM011 (número de acceso AF195130) dentro de la secuencia de un intrón del gen ELA-DMA (L100061035), a COR112 en una región no codificante entre los pseudogenes DRB1-4 (LOC100146181; posición 33.246.36933.251.023) y DOB (LOC100060663; posición 33.302.539-33.307.437pb). Se desarrolló la metodología para detectar el microsatélite del gen ELA-DRB2 (STR2), localizado en el intrón 2 del gen ELA-DRB2 (LOC100052310; posición33.193.749-33.193.985pb). La representación esquemática del mapa de los tres STRs en relación al MHC se muestra en la Figura 1.

\section{Tipificación y polimorfismo de los STRs}

La tipificación de los microsatélites fue realizada en un total de 30 caballos de raza Árabe usando tres loci STRs descritos en el apartado Materiales y Métodos. Se detectó un número variable de alelos en cada microsatélite, con 2 alelos en STR2, 5 alelos en COR112 y 7 en UM011 (Tabla 1). Las distribución de frecuencias alélicas, así como los valores de diversidad genética (He y Ho), PIC y HWE se detallan en la Tabla 1. Los tres STRs se encontraron en equilibrio de Hardy-Weinberg $(p<0,05)$ y los valores de diversidad genética variaron entre $\mathrm{He}=35$ y $72 \%$. COR112 y UM011 mostraron valores de contenido informativo del polimorfismo (PIC) de 64 y $69 \%$, respectivamente. La

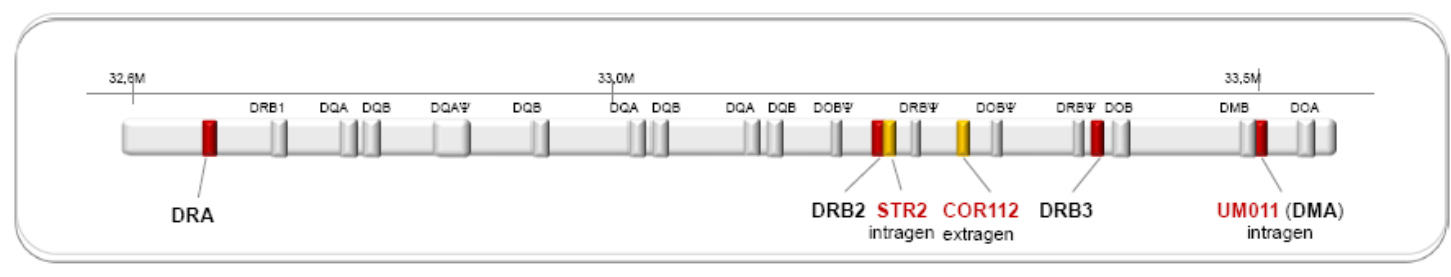

Figura 1. Mapa esquemático que representa la región de clase II del ELA en el Eca20. Se señalaron en color amarillo los marcadores STRs utilizados (microsatélites), indicando su localización con respecto a las secuencias codificantes (intragen/extragen). Se indican también genes y pseudogenes de clase ll y su posición dentro de la región cromosómica. 
distribución de los alelos mostró uno o dos alelos más frecuentes y varios alelos con frecuencias menores. Se investigó el desequilibrio de ligamiento entre los tres marcadores STRs, con valores altamente significativos entre CORR112 y UM011. los haplotipos A, C, D y E presentaron una frecuencia de $12 \%$. Cuatro haplotipos (F, G, H, J) mostraron frecuencias entre 1,7-4,2\%. Cinco de los 10 haplotipos son compartidos y cada grupo familiar presentó entre 1 y 5 haplotipos exclusivos (Tabla 3 ).

Tabla 1. Distribución de los alelos de los tres STRs y los valores de diversidad genética estimados para He y Ho.

\begin{tabular}{|c|c|c|c|c|c|}
\hline \multicolumn{6}{|c|}{ Locus STR } \\
\hline \multicolumn{2}{|c|}{ STR2 } & \multicolumn{2}{|c|}{ COR112 } & \multicolumn{2}{|c|}{ UM011 } \\
\hline Alelo & Frecuencia & Alelo & Frecuencia & Alelo & Frecuencia \\
\hline 234 & 0,776 & 245 & 0,121 & 160 & 0,017 \\
\hline \multirow[t]{6}{*}{236} & 0,224 & 247 & 0,362 & 162 & 0,086 \\
\hline & & 253 & 0,086 & 164 & 0,138 \\
\hline & & 255 & 0,397 & 166 & 0,121 \\
\hline & & 257 & 0,034 & 168 & 0,138 \\
\hline & & & & 172 & 0,034 \\
\hline & & & & 178 & 0,466 \\
\hline na & 2 & & 5 & & \\
\hline $\mathrm{He}$ & 0,354 & & 01 & & \\
\hline $\mathrm{Ho}$ & 0,448 & & 89 & & \\
\hline EHW & 0,288 & & 52 & & \\
\hline PIC & 0,287 & & 34 & & \\
\hline
\end{tabular}

Tabla 2. Estimación del desequilibrio de ligamiento entre los marcadores STRs COR112, UM011 y STR2 ${ }^{* * *}$ altamente significativo con $\alpha \leq 0,05$.

\begin{tabular}{cccc}
\hline Locus 1 & Locus 2 & P-Value & S.E. \\
\hline STR2 & COR112 & 0,0915 & 0.003904 \\
STR2 & UMO11 & 0,6106 & 0.009589 \\
COR112 & UMO11 & $\mathbf{0 , 0 0 0 0}^{\text {*** }}$ & 0.000000 \\
\hline
\end{tabular}

\section{Identificación de haplotipos}

Los marcadores microsatélites localizados en ligamiento en la región cromosómica del ELA se consideraron en conjunto para determinar las combinaciones haplotípicas presentes en toda la muestra analizada. El análisis con el algoritmo PHASE permitió identificar la presencia de 24 haplotipos probables con frecuencias entre $0,04 \%$ y $19,6 \%$. Con el fin de verificar la presencia de cada combinación haplotípica en los caballos analizados, se realizó un análisis de segregación en tres grupos familiares compuestos por dos y tres generaciones. Se identificaron un total de 10 haplotipos segregantes en las tres familias analizadas. Para identificarlos y realizar las comparaciones se asignó una letra mayúscula a cada haplotipo (A-K). El haplotipo más frecuente fue B $(20 \%)$, en tanto que

\section{Discusión}

Los caballos de raza Árabe tipificados mostraron un grado apreciable de polimorfismo y de diversidad genética, evidenciado por valores de heterocigosis $\geq 70 \%$ de los marcadores microsatélites analizados, y entre 2 y 7 alelos. Algunos alelos fueron raros (frecuencia $<3 \%$ ) y se observaron solamente en unos pocos animales, sin una contribución mayor a la identificación de los haplotipos.

El STR2 presentó solamente dos alelos y un valor de heterocigosis esperada de $35 \%$. Dado que se encuentra ubicado en el segundo intrón 2 del gen ELA-DRB2, es probable que se encuentre estrechamente ligado a algunos alelos del exón 2 de este gen, por lo que la presencia de más alelos estaría condicionada por el alelo del exón 2 presente en cada individuo. Sin embargo, el marcador STR2, así como UM011 y COR112, evidenciaron equilibrio de EHW en la población analizada.

El nivel de variación genética observado en la región de clase II del ELA en los caballos de raza Árabe permitió inferir la existencia de numerosas combinaciones haplotípicas. La estimación estadística de las diferentes combinaciones posibles de todos los alelos de los STRs permitió identificar veinticuatro haplotipos diferentes con frecuencias de entre $0,1 \%$ y $20 \%$. Los haplotipos con frecuencias menores al $0,5 \%$ no fueron considerados en el análisis. Para validar esta premisa, se realizó un análisis de segregación familiar, teniendo 
Tabla 3. Combinaciones haplotípicas de los microsatélites del ELA inferidos mediante el análisis con el algoritmo PHASE en los caballos de raza Árabe analizados en este trabajo. Se indica la frecuencia estimada para cada uno de los 10 haplotipos más frecuentes (designados por letras mayúsculas) que fueron también verificados por segregación familiar en tres grupos familiares (I, II y III); además, se indica el número de individuos que se asociaron con cada haplotipo.

\begin{tabular}{ccccccc}
\hline Haplotipo & & STR/alelo & & Frecuencia & Familia & N \\
\hline A & STR2-234 & COR112-245 & UMO11-178 & 0,1196 & I, II, III & 7 \\
B & STR2-236 & COR112-255 & UMO11-178 & 0,1966 & I, II & 10 \\
C & STR2-234 & COR112-255 & UMO11-178 & 0,1244 & II, III & 9 \\
D & STR2-234 & COR112-247 & UMO11-166 & 0,1197 & I, II, III & 7 \\
E & STR2-234 & COR112-247 & UMO11-168 & 0,1202 & I, II & 7 \\
F & STR2-234 & COR112-247 & UMO11-172 & 0,0345 & III & 2 \\
G & STR2-234 & COR112-255 & UMO11-162 & 0,0424 & II & 3 \\
H & STR2-234 & COR112-257 & UMO11-160 & 0,0172 & II & 1 \\
J & STR2-234 & COR112-253 & UMO11-164 & 0,0347 & II & 2 \\
K & STR2-234 & COR112-247 & UMO11-164 & 0,0389 & I & 1 \\
\hline
\end{tabular}

en cuenta los registros de pedigree en el Stud Book Argentino (http://www.studbook.com.ar/) de cada individuo de los grupos familiares incluidos en el estudio.

Mediante el análisis de segregación familiar, 10 de los 24 haplotipos estimados pudieron identificarse en los grupos familiares de caballos de raza Árabe. Cada grupo estaba compuesto por 5-7 individuos, y los mencionados haplotipos estaban presentes en distintos individuos de cada generación incluida en el pedigree. Como ejemplo, podemos mencionar el haplotipo $A$, presente en las tres familias. En la familia $1(n=7)$, el haplotipo A estaba presente en la abuela 76, en la hija 75 y en el nieto 167 (Figura 2). En esta familia, compuesta por 7 individuos representando tres generaciones, se detectaron 5 haplotipos diferentes (A, B, D, E, K). Cada uno de ellos pudo ser verificado a través del análisis de segregación familiar (Figura 2). Los restantes haplotipos se encontraron asociados a uno o pocos individuos y no se verificaron por segregación familiar.

En todas las familias se hallaron entre 4 y 7 haplotipos diferentes; algunos eran compartidos por todas las familias (haplotipos A, D), en tanto que otros estaban presentes solamente en uno o dos grupos familiares (haplotipos B, C, E).

La presencia de combinaciones haplotípicas compartidas entre diferentes grupos familiares indicaría la posible presencia de haplotipos ancestrales. Dado que la raza árabe se caracteriza por un registro genealógico cerrado y la presencia de padrillos fundadores y líneas maternas bien definidas en el origen de la raza, es probable que estos haplotipos pertenezcan a haplogrupos mayores. Por tal motivo, la ancestría podría comprobarse adicionando al análisis y caracterización de los haplotipos algunos marcadores de genes estructurales, y evaluando las distancias mínimas necesarias entre marcadores para detectar desequilibrio de ligamiento (DL) y, por lo tanto, definir y validar los haplotipos segregantes con posible relevancia funcional.

EI DL altamente significativo dentro del genoma equino entre razas, probablemente refleje la reciente divergencia entre las mismas y el hecho de compartir fundadores (Wade et al., 2009). Mientras que algunos haplotipos del ELA son compartidos entre razas, estudios previos describen diferencias entre razas en la frecuencia de varios haplotipos del ELA, incluyendo la aparente ausencia de algunos haplotipos en varias razas. Además, se hallaron características similares en los haplotipos de microsatélites definidos particularmente en las razas Thoroughbreds y Standardbreds (Tseng et al., 2011). Usando un conjunto de microsatélites, incluyendo los aquí utilizados, Tseng et al. (2010) demostraron la presencia de 19 haplotipos en caballos de las razas Standardbreds y Thoroughbreds, en una muestra de alrededor de cien caballos de cada una. Los resultados reportados ponen en evidencia numerosos haplotipos únicos, aun en grupos familiares reducidos y con tres marcadores ligados dentro de la región de clase II. Además, observaron que los haplotipos de microsatélites del MHC se dividen a lo largo de las líneas raciales. Cabe señalar que, por su historia documentada, la raza Thoroughbred se originó hace aproximadamente 300 años en Inglaterra, a partir de un pequeño grupo que, se cree, era producto de la cruza entre caballos Árabes y caballos nativos de Inglaterra (Willett, 1970) y que la raza American Standardbred se originó en los Estados Unidos hace aproximadamente 150 años, e incluye caballos 


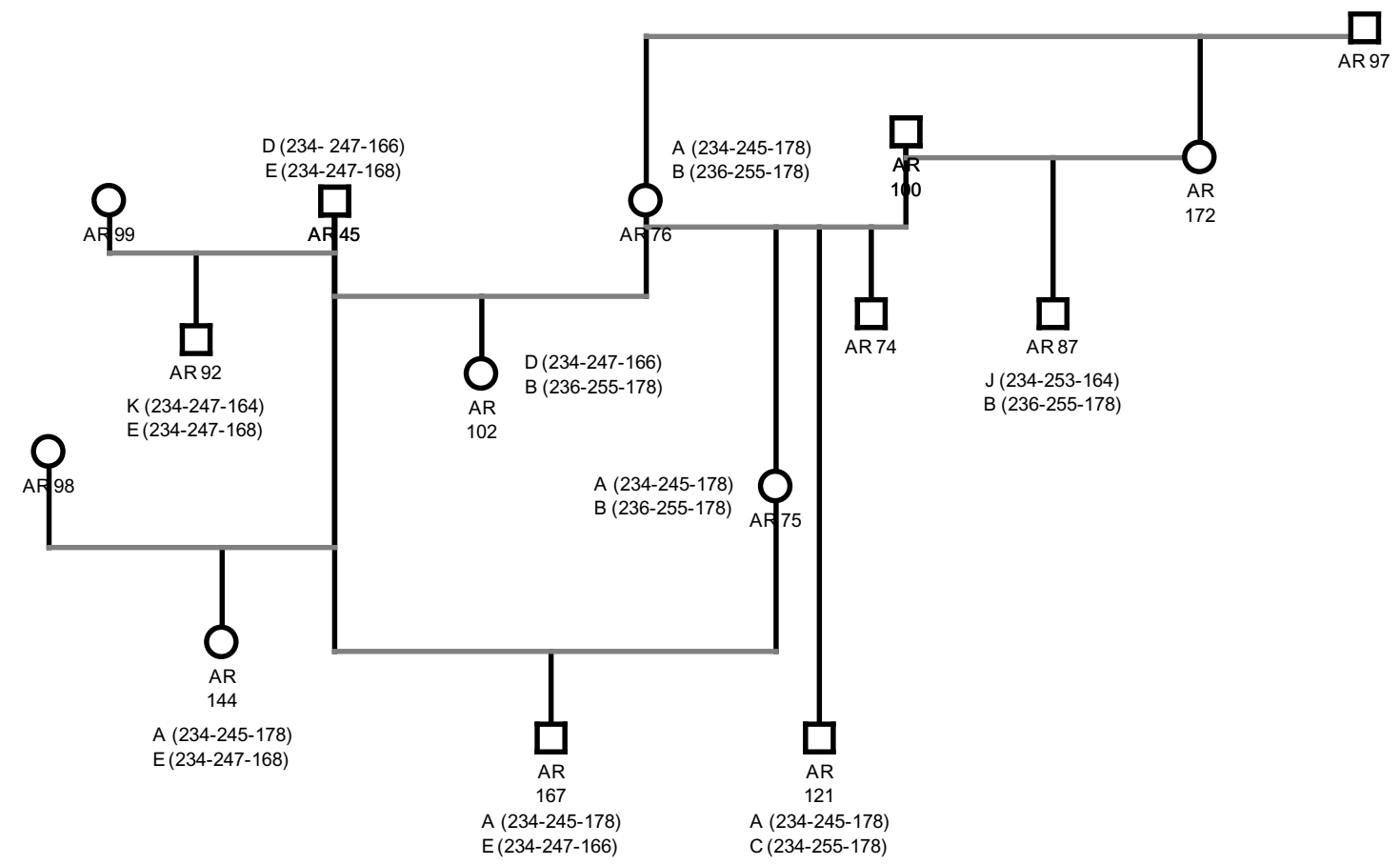

Figura 2. Pedigree que demuestra la segregación de los haplotipos conformados por los marcadores STR2, COR112 y UMO11 en una línea familiar de caballos de raza Árabe. La representación del pedigree se realizó con el programa Genopro®2016 Versión 3.0.0.

Thoroughbred entre sus fundadores. Sin embargo, estos autores señalan que ninguno de los haplotipos descritos en Thoroughbreds y Standardbreds estaba asociado con los caballos de raza Árabe, aun cuando se demostró que estos eran portadores de algunos de los serotipos del ELA detectados en estudios previos (Crepaldi et al., 1986). En el futuro será de interés determinar la distribución de los haplotipos de los microsatélites entre razas equinas.

La metodología de tipificación mediante el uso de microsatélites del MHC reportada en este estudio permite una identificación rápida de los haplotipos del MHC equino en la mayoría de los caballos/familias analizados. Constituye un complemento de utilidad para una diferenciación rápida y adecuada de diferentes haplotipos equinos, como alternativa a la secuenciación y/o determinación alelo específica. La cantidad de haplotipos identificada permitió expandir la valoración de la diversidad del MHC equino y brinda herramientas adicionales para el análisis de diversidad genética del MHC de los caballos, haciendo posible analizar la estructura poblacional, el desequilibrio de ligamiento y la asociación con caracteres de producción y en estudios genético-poblacionales relacionados con la inmunidad.

\section{Agradecimientos}

Los autores desean agradecer a los criadores de caballos de la raza Árabe por aportar muestras para este estudio. Este trabajo fue financiado mediante el Proyecto PICT2012-2610 (ANPCyT) y el Proyecto Acreditado 11/V206 del Programa Nacional de Inventivos.

\section{Conflicto de intereses}

Todos los autores declaran que no existe conflicto de intereses, incluyendo las relaciones financieras, personales o de otro tipo con otras personas u organizaciones que pudieran influir de manera inapropiada en el trabajo.

\section{Bibliografía}

Ansari HA, Hediger R, Fries RY, Stranzinger G. 1988. Chromosomal localization of the major histocompatibility complex of the horse (ELA) by in situ hibridization. Immunogenetics. 28:362-4.

Antczak DF. The major histocompatibility complex of the horse. En: Plowright W, Rossdale PD, Wade JF. 1992. Equine Infectious Diseases VI, "Proceedings of the Sixth International Conference" 7th-11th July, 1991. New York, R\&W Publications, pp. 99-112.

Azor PJ, Mercedes V, Gómez MD, Goyache F, Molina A. 2007. Genetic characterization of the Spanish Trotter horse breed using microsatellite markers. Genetics and Molecular Biology. 30(1):37-42.

Bastos Silveira C, Luís C, Ginja C, Gama LT, Oom MM. 2009. Genetic variation in BoLA microsatellite loci in Portuguese cattle breeds. Animal Genetics. 40(1):101-5.

Brinkmeyer-Langford CL, Cai JJ, Gill CA Skow LC. 2013. Microsatellite variation in the equine MHC. Animal Genetics. 44(3):267-75.

Chardon P, Renard CY, Vaiman M. 1999. The major histocompatibility complex in swine. Immunological Reviews. 167:179-92. 


\section{Sadaba SA et al.}

Cox RA. 1968. Methods in Enzymology (Grossmann L \& Moldave E Eds.) Vol. 12, Part B. Orlando, Academic Press, pp. 120-9.

Crepaldi T, Crump A, Newman M, Ferrone S, Antczak DF. 1986. Equine T-lymphocytes express MHC class II antigens. Journal of Immunogenetics. 13(4):349-60.

Hedrick, P.W. 1994. Evolutionary genetics of the major histocompatibility complex. The American Naturalist. 143(6):945-964.

Hughes AL, Nei M. 1992. Models of host-parasite interaction and MHC polymorphism. Genetics.132(3):863.

Ellis SA, Ballingall KT. 1999. Cattle MHC: evolution in action? Immunology Reviews. 167:159-68.

Kalemkerian PB, Metz GE, Peral-García P, López-Gappa J, Echeverría MG, Giovambattista G, Díaz S. 2011. Eca20 microsatellite polymorphisms in equine viral arteritis-infected horses from Argentina. Tissue Antigens. 78(4):281-4.

Lazary S, Gerber H, Glatt PA, Straub R. 1985. Equine leukocyte antigens in sarcoid-infected horses. Equine Veterinary Journal. 14:283-6.

Lazary S, Antczak DF, Bailey E, Bell TK, Bernoco D, Byrns G, McClure JJ. 1988. Joint Report of the Fifth International Joint Report of the Fifth International Workshop on Lymphocyte Alloantigens of the Horse, Baton Rouge, Louisiana, 31 October-1 November 1987. Animal Genetics. 19(4):447-56.

Lewin HA, Russell GC, Glass EJ. 1999. Comparative organization and function of the major histocompatibility complex of domesticated cattle. Immunological Reviews. 167:145-58.

Maillard JC, Chantal I, Berthier D, Thevenon S, Sidibe I, Razafindraibe H. 2002. Molecular immunogenetics in susceptibility to bovine dermatophilosis: a candidate gene approach and a concrete field application. Annals of the New York Academy of Science. 969:92-6.

Meagher S, Potts WK. 1997. A microsatellite-based MHC genotyping system for house mice (Mus domesticus). Hereditas. 127:75-82.

Meredith D, Elser AH, Wolf B, Soma LR, Donawick WJ, Lazary S. 1986. Equine leukocyte antigens: relationships with sarcoid tumors and laminitis in two pure breeds. Immunogenetics. 23:221-5.

Meyer AH, Valberg SJ, Hillers KR, Schweitzer JK, Mickelson JR. 1997. Sixteen new polymorphic equine microsatellites. Animal Genetics. 28:69-70.

Rousset F. 2008. Genepop'007: a complete reimplementation of the Genepop software for Windows and Linux. Molecular Ecology Resources. 8:103-6.

Santucci F, Ibrahim KM, Bruzzone A, Hewit GM. 2007. Selection on MHC-linked microsatellite loci in sheep populations. Heredity. 99(3):340-8.

Sharif S, Mallard BA, Wilkie BN, Sargeant JM, Scott HM, Dekkers JC, Leslie KE. 1998. Associations of the bovine major histocompatibility complex DRB3 (BoLA-DRB3) alleles with occurrence of disease and milk somatic cell score in Canadian dairy cattle. Animal Genetics. 29(3):185-93.

Swinburne LS, Meadows JR, Broström H, Eriksson S, Fikse WF, Frey R, Sundquist M, Tseng CT, Mikko S, Lindgren G. 2012. The same ELA class II risk factors confer equine insect bite hypersensitivity in two distinct populations. Immunogenetics. 64(3):201-8.

Tseng CT, Miller D, Cassano J, Bailey E, Antczak DF. 2010. Identification of equine major histocompatibility complex haplotypes using polymorphic microsatellites. Animal Genetics. 41:150-3.

Wade CM, Giulotto E, Sigurdsson S, Zoli M, Gnerre S, Imsland F, Swinburne JE. 2009. Genome sequence, comparative analysis, and population genetics of the domestic horse. Science. 326(5954):865-7.

Wagner JL, Burnett RC, DeRose SA, Francisco LV, Storb R, Ostrander EA. 1996. Histocompatibility testing of dog families with highly polymorphic microsatellite markers. Transplantation. 62(6):876-7.

Willett P. 1970. The Thoroughbred. London, Weidenfeld \& Nicolson Ltd. 